\title{
A Termination Criterion for Iterative Methods Used to Find the Zeros of Polynomials
}

\author{
By Masao Igarashi
}

\begin{abstract}
A new criterion for terminating iterations when searching for polynomial zeros is described. This method does not depend on the number of digits in the mantissa; moreover, it can be used to determine the accuracy of the resulting zeros. Examples are included.
\end{abstract}

1. Introduction. This section discusses some of the better known termination criteria as applied to Newton-Raphson's method. This method iterates according to the following procedure:

$$
x_{k+1}=x_{k}-f\left(x_{k}\right) / f^{\prime}\left(x_{k}\right),
$$

where $f(x)$ is a polynomial of the form

$$
f(x)=a_{0} x^{n}+a_{1} x^{n-1}+\cdots+a_{n-1} x+a_{n} \quad\left(a_{0} a_{n} \neq 0.0\right) .
$$

Several well-known criteria for terminating the calculations are ([1], [2], [3], [4]):

$$
\begin{gathered}
\left|x_{k+1}-x_{k}\right| \leqslant \operatorname{EPS}\left|x_{k+1}\right|, \\
\left|f\left(x_{k+1}\right)\right| \leqslant \operatorname{EPS}\left(\sum_{j=0}^{n}\left|a_{j} x_{k+1}^{n-j}\right|\right), \\
\left|f\left(x_{k+1}\right)\right| \leqslant \operatorname{EPS}\left(\max _{j}\left|a_{j} x_{k+1}^{n-j}\right|\right) \quad(j=0,1,2, \ldots, n),
\end{gathered}
$$

where EPS is a constant which depends on the number of digits. For a computer performing floating-point arithmetic with a 24-bit mantissa, the value of EPS is nearly equal to $2^{-24}$. Criterion 1 ) is simple and effective if $x_{k}$ approaches a sufficiently isolated zero, while ineffective if $x_{k}$ approaches multiple or clusters of zeros.

When calculating $f\left(x_{k}\right)$ by floating-point arithmetic, the number of significant digits decreases as $x_{k}$ approaches a zero. Hence, procedure (1) should terminate if $f\left(x_{k+1}\right)$ has no significant digits. Criteria 2) and 3) are based on this principle. That is, the right side of these relations sets an upper bound on the calculation error of $f\left(x_{k+1}\right)$. When using criteria 2) and 3), it is difficult to determine the value of $E P S$ adequately. For example, assigning EPS a value of $2^{-24}$, when using criterion 2) on a floating-point binary arithmetic machine with a 24-bit mantissa, results in an over-estimation [4]. Using the same value on a floating-point hexadecimal arithmetic

Received April 26, 1982; revised August 11, 1982 and March 17, 1983.

1980 Mathematics Subject Classification. Primary 65G05, 65H05.

Key words and phrases. Algebraic equation, zeros of polynomials, round-off errors. 
machine with a 24-bit mantissa results in an under-estimation, because a few leading bits of the mantissa are sometimes lost in the calculation on a hexadecima: arithmetic machine.

2. Termination Criterion. In this section we evaluate $f(x)$ using two different procedures, and describe a termination criterion that uses the difference in the resulting values.

Procedure I. We evaluate $f(x)$ by one of the usual methods, such as Horner's, and let that value be $A(x)$.

Procedure II. Next, we evaluate $G(x)$ as given by

$$
\begin{array}{r}
G(x)=(n-1) a_{0} x^{n}+(n-2) a_{1} x^{n-1}+\cdots+a_{n-2} x^{2}-a_{n} \\
\left(=x f^{\prime}(x)-f(x)\right)
\end{array}
$$

followed by $x f^{\prime}(x)$, and finally $x f^{\prime}(x)-G(x)$, whose value we represent as $B(x)$.

If $x$ lies near a zero of (2), then the computed value $A(x)$ has more significant digits than the computed $B(x)$, because the relation $\left|x f^{\prime}(x)\right|>|f(x)|$ always holds true near a zero of (2). Therefore, the value for $f(x)$ obtained by Procedure $I$ is somewhat more accurate than that obtained by Procedure II. However, since the exponent of $A(x)$ agrees with that of $B(x)$, we may conclude that as $x$ comes sufficiently close to a zero of (2), both $A(x)$ and $B(x)$ cease to have any accurate digits, and the two values differ.

We now present the following termination criterion based on the above discussion:

$$
\left|A\left(x_{k}\right)-B\left(x_{k}\right)\right| \geqslant \min \left(\left|A\left(x_{k}\right)\right|,\left|B\left(x_{k}\right)\right|\right) .
$$

Next, some detailed characteristics of the criterion will be considered.

C-1) If $A\left(x_{k}\right)=0.0$ or $B\left(x_{k}\right)=0.0$, then the iteration terminates.

C-2) If $A\left(x_{k}\right) B\left(x_{k}\right)<0.0$, then the iteration terminates.

C-3) If $A\left(x_{k}\right) B\left(x_{k}\right)>0.0$, then (3) is replaced by

$$
\frac{\left|A\left(x_{k}\right)-B\left(x_{k}\right)\right|}{\min \left(\left|A\left(x_{k}\right)\right|,\left|B\left(x_{k}\right)\right|\right)} \geqslant 1 .
$$

This means that the iteration will terminate if $2\left|A\left(x_{k}\right)\right| \leqslant\left|B\left(x_{k}\right)\right|$ or $2\left|B\left(x_{k}\right)\right| \leqslant$ $\left|A\left(x_{k}\right)\right|$.

3. Application and Comparison. The above criterion is easily applied to other methods, such as Durand-Kerner's method [5] and Aberth's [6] method. Furthermore it is possible to estimate the accuracy of the result by using the values $A(x)$ and $B(x)$ [4]. That is, if $x_{k+1}$ satisfies criterion (3), then the number of leading digits of $x_{k}-A\left(x_{k}\right) / f^{\prime}\left(x_{k}\right)$ and $x_{k}-B\left(x_{k}\right) / f^{\prime}\left(x_{k}\right)$ which are in agreement is nearly equal to the number of accurate digits. In Example 4 (Table 1), for instance, if we take $x_{0}=1.277$ as an initial approximation, then we have the following approximations.

$$
\begin{aligned}
& x_{0}-A\left(x_{0}\right) / f^{\prime}\left(x_{0}\right)=0.127172646983610 \mathrm{D}+01, \\
& x_{0}-B\left(x_{0}\right) / f^{\prime}\left(x_{0}\right)=0.127172697536861 \mathrm{D}+01,
\end{aligned}
$$


where the value for the left side of $(4)$ is $0.13 \mathrm{D}-02$. The leading digits which are in agreement $(0.1271726 \mathrm{D}+01)$ are accurate digits, and the leading digits up to the second decimal place $(0.12 \mathrm{D}+01)$ agree with the solution. As the iteration continues, the number of accurate digits decreases, and after five iterations the following approximations are obtained:

$$
\begin{aligned}
& x_{5}-A\left(x_{5}\right) / f^{\prime}\left(x_{5}\right)=0.126008718707480 \mathrm{D}+01, \\
& x_{5}-B\left(x_{5}\right) / f^{\prime}\left(x_{5}\right)=0.126015476865675 \mathrm{D}+01,
\end{aligned}
$$

where the left side of (4) evaluates to $0.38 \mathrm{D}+01$. This shows that the number of leading digits which are in agreement $(=0.1260 \mathrm{D}+01)$ is nearly equal to the number of significant digits in the true zero.

\section{TABLE 1}

1. $(x-12.5)^{3}=x^{3}-37.5 x^{2}+468.75 x-1953.125$

2. $(x-1.20)(x-1.21)(x-1.22)(x-1.23)=x^{4}-4.86 x^{3}+8.85701 x^{2}-7.173846 x+2.178812$

3. $(x-1)(x-2) \ldots(x-6)=x^{6}-21 x^{5}+175 x^{4}-735 x^{3}+1624 x^{2}-1764 x+720$

4. $(x-1.20)(x-1.21) \ldots(x-1.26)=x^{7}-8.61 x^{6}+31.7695 x^{5}-65.121735 x^{4}+80.08914424 x^{3}$ $-59.0953690404 x^{2}+24.2237621 x-4.2553354536$

5. $(x+1.5)\left(x^{2}+3 x+4\right)\left(x^{2}+2 x+2\right)\left(x^{2}+x+1\right)=x^{7}-3 \cdot 5 x^{6}+5 x^{5}-2 x^{4}+4 \cdot 5 x^{3}-15 x^{2}+17 x-12$

6. $(x-8-9 i)^{4}(x-8+9 i)^{4}=x^{8}-64 x^{7}+2116 x^{6}-44224 x^{5}+637126 x^{4}-6412480 x^{3}$ $+44488900 x^{2}-195112000 x+442050625$

7. $x^{10}+2 x^{9}+6 x^{8}+8 x^{7}+121046 x^{6}+242076 x^{5}+484144 x^{4}+484136 x^{3}+3662549361 x^{2}$ $+7324130450 x+7324130450$

8. $x^{10}-206 x^{9}+10800 x^{8}-21500 x^{7}+1060 x^{6}-21.1 x^{5}+0.211 x^{4}-0.00106 x^{3}+0.00000217 x^{2}$ $-0.000000000155 x-0.000000000000000114$

9. $x^{12}-78 x^{11}+1001 x^{10}-5005 x^{9}+12870 x^{8}-19448 x^{7}+18564 x^{6}-11628 x^{5}+4845 x^{4}-1330 x^{3}$ $+231 x^{2}-23 x+1$

10. $x^{14}-2.5 x^{12}+2.375 x^{10}-1.086309523 x^{8}+0.249627956 x^{6}-0.02754667202 x^{4}$ $+0.001130581327 x^{2}-0.0001025063224$

11. $x^{20}-200 x^{18}+6600 x^{16}-84480 x^{14}+549120 x^{12}-2050048 x^{10}+4659200 x^{8}-6553600 x^{6}$ $+5570560 x^{4}-2621440 x^{2}+524288$

12. $x^{29}+x^{28}+x^{27}+\ldots+x^{2}+x+1$ 
TABLE 2

Comparison of 2) with (3) about the iteration times

\begin{tabular}{|l|l|llllllllllllll|}
\hline & \multicolumn{2}{|c}{ No. } & 2 & 2 & 3 & 4 & 5 & 6 & 7 & 8 & 9 & 10 & 11 & 12 \\
\hline \multirow{3}{*}{ D-K. } & $21-52$ & 34 & 30 & 30 & 55 & 25 & 56 & 18 & 106 & 95 & 31 & 57 & 108 \\
& $21-56$ & 45 & 31 & 30 & 58 & 25 & 59 & 24 & 107 & 96 & $*$ & 58 & $*$ \\
& $(3)$ & 35 & 30 & 31 & 58 & 29 & 58 & 18 & 110 & 96 & 31 & 57 & 108 \\
\hline \multirow{5}{*}{ Ab. } & $21-52$ & 20 & 18 & 17 & 29 & 14 & 31 & 12 & 58 & 51 & 16 & 30 & 54 \\
& $21-56$ & 26 & 18 & 29 & 34 & 15 & 35 & 19 & 59 & 52 & $*$ & 31 & $\vdots$ \\
& $(13)$ & 21 & 18 & 18 & 31 & 12 & 32 & 12 & 60 & 52 & 16 & 31 & 54 \\
\hline
\end{tabular}

The symbol '**' means the iteration was not terminated, and

2) -52 means $\left.E P S=2^{-52} ; 2\right)-56, E P S=2^{-56}$.

Next, we will compare the number of iterations necessary for termination when using criteria 2) and 3). Table 1 gives the polynomials used in this comparison, and Table 2 lists the results. If EPS is $2^{-56}$ in criterion 2), some iterations do not terminate. If $E P S$ is assigned a value of $2^{-52}$, all iterations terminate, and the number of iterations required is nearly the same as for criterion (3). Thus, with an $E P S$ value of $2^{-52}$, criterion 2) yields accuracy similar to criterion (3); however, with criterion (3) we are not burdened with the task of finding an appropriate EPS value.

Smith's error estimation $(=S(I))[7]$ is sometimes used to investigate the accuracy of approximated zeros. However, this method sometimes displays instability, due to the fact that round-off errors occurring near zeros may cause $\left|f\left(x_{k}\right)\right|$ to take on a smaller than true value (see Table 5, No. 2).

4. Numerical Examples and Remarks. We now present the results of some numerical experiments to illustrate the efficiency of criterion (3). Newton-Raphson's, Durand-Kerner's and Aberth's methods are employed. The machine used is a HITAC L340, floating-point hexadecimal arithmetic using a 56-digit mantissa.

TABLE 3-1

$R(I)$ and $N$ for Durand-Kerner's method

\begin{tabular}{|l|rrrrrrrrrrrr|c|}
\hline No. & 1 & 2 & 3 & 4 & 5 & 6 & 7 & 8 & 9 & 10 & 11 & 12 & Total \\
\hline$I \leq R(I)<2$ & 3 & 2 & 0 & 1 & 1 & 4 & 0 & 0 & 2 & 0 & 6 & 3 & 22 \\
\hline $2 \leq R(I)<4$ & 0 & 0 & 1 & 4 & 1 & 2 & 2 & 3 & 2 & 3 & 3 & 1 & 22 \\
\hline $4 \leq R(I)$ & 0 & 2 & 5 & 2 & 5 & 2 & 8 & 7 & 8 & 11 & 11 & 25 & 86 \\
\hline$N$ & 35 & 30 & 31 & 58 & 29 & 58 & 18 & 110 & 96 & 31 & 57 & 108 & 661 \\
\hline
\end{tabular}

TABLE 3-2

$R(I)$ and $N$ for Aberth's method

\begin{tabular}{|c|rrrrrrrrrrrr|c|}
\hline No. & 1 & 2 & 3 & 4 & 5 & 6 & 7 & 8 & 9 & 10 & 11 & 12 & Total \\
\hline $1 \leq R(I)<2$ & 1 & 0 & 0 & 0 & 0 & 2 & 1 & 1 & 1 & 3 & 2 & 0 & 11 \\
\hline $2 \leq R(I)<4$ & 1 & 0 & 1 & 6 & 0 & 4 & 0 & 3 & 3 & 0 & 2 & 1 & 21 \\
\hline $4 \leq R(I)$ & 1 & 4 & 5 & 1 & 7 & 2 & 9 & 6 & 8 & 11 & 16 & 28 & 98 \\
\hline$N$ & 21 & 28 & 18 & 31 & 12 & 32 & 12 & 60 & 52 & 16 & 31 & 54 & 359 \\
\hline
\end{tabular}


TABLE 4

Numerical solutions obtained by Durand-Kerner's method

\begin{tabular}{|c|c|c|c|c|}
\hline & Approx & al. Ions & & \\
\hline No. & Real part & Imaginary part & $S(I)$ & $R(I)$ \\
\hline 4 & $\begin{array}{l}0.1260022530571170+01 \\
0.12600 \underline{44935787260}+01\end{array}$ & $\begin{array}{l}-0.1614486101142410-05^{\circ} \\
-0 . \underline{1737394493684690}-05\end{array}$ & $0.330-04$ & $0.250+01$ \\
\hline & $\begin{array}{l}0.1240154328156920+01 \\
0.1239713486218810+01\end{array}$ & $\begin{array}{l}0.1418692117313880-03 \\
0.2315357904719180-03\end{array}$ & $0.140-02$ & $0.14 D+01$ \\
\hline & $\begin{array}{l}0.122026142817432 D+01 \\
0.121999543294664 D+01\end{array}$ & $\begin{array}{l}0.290475473912133 D-05 \\
0.507746249261375 D-05\end{array}$ & $0.170-03$ & $0.82 D+01$ \\
\hline & $\begin{array}{l}0.120001327505341 D+01 \\
0.120001832390272 D+01\end{array}$ & $\begin{array}{l}-0.116280271027772 D-05 \\
-0.116643564166874 D-05\end{array}$ & $0.380-04$ & $0.27 D+01$ \\
\hline & $\begin{array}{l}0.12 C .997590132129 D+01 \\
0.121005443136218 D+01\end{array}$ & $\begin{array}{l}0.177938570470326 \mathrm{D}-04 \\
0.155796570015701 \mathrm{D}-05\end{array}$ & $0.13 D-03$ & $0.53 D+01$ \\
\hline & $\begin{array}{l}0.122956622901893 D+01 \\
0.122991616096711 D+01\end{array}$ & $\begin{array}{l}0.165007368735400 \mathrm{D}-04 \\
0.249230062104850 \mathrm{D}-04\end{array}$ & $0.63 D-03$ & $0.280+01$ \\
\hline & $\begin{array}{l}0.124980042353348 D+01 \\
0.124983170585586 D+01\end{array}$ & $\begin{array}{l}0.123948865122464 D-04 \\
0.199767649929021 D-04\end{array}$ & $0.100-02$ & $0.28 D+01$ \\
\hline 10 & $\begin{array}{l}0.949136366576219 \mathrm{D}+00 \\
0.949136366576207 \mathrm{D}+00\end{array}$ & $\begin{array}{l}-0.546700880998977 D-14 \\
-0.546671513097082 D-14\end{array}$ & $0.11 D-12$ & $0.430+01$ \\
\hline & $\begin{array}{l}0.760489880828673 D+00 \\
0.760489880828684 D+\infty\end{array}$ & $\begin{array}{l}0.625088489166552 \mathrm{D}-01 \\
0.625088489166436 \mathrm{D}-01\end{array}$ & $0.38 D-13$ & $0.11 D+02$ \\
\hline & $\begin{array}{l}0.496971875530099 D+\infty 0 \\
0.496971875530098 D+\infty\end{array}$ & $\begin{array}{l}0.1506083606481350+00 \\
0.150608360648136 D+00\end{array}$ & $0.180-14$ & $0.180+02$ \\
\hline & $\begin{array}{l}0.185435167382210 D+\infty \\
0.1854351673822110+\infty\end{array}$ & $\begin{array}{l}0.1831714822278000+\infty 0 \\
0.1831714822278000+\infty 0\end{array}$ & $0.170-15$ & $0.570+01$ \\
\hline & $\begin{array}{l}-0.185435167382210 D+\infty \\
-0.1854351673822100+\infty\end{array}$ & $\begin{array}{l}0.1831714822278000+\infty \\
0.183171482227800 D+\infty\end{array}$ & $0.170-15$ & $0.570+01$ \\
\hline & $\begin{array}{l}-0.496971875530099 D+\infty \\
-0.4969718755300970+\infty\end{array}$ & $\begin{array}{l}0.150608360648135 D+00 \\
0.150608360648134 D+00\end{array}$ & $0.180-14$ & $0.180+02$ \\
\hline & $\begin{array}{l}-0.760489880828673 \mathrm{D}+00 \\
-0.760489880828685 \mathrm{D}+00\end{array}$ & $\begin{array}{l}0.625088489166553 \mathrm{D}-01 \\
0.625088489166335 \mathrm{D}-01\end{array}$ & $0.590-13$ & $0.310+01$ \\
\hline & $\begin{array}{l}-0.949136366576213 D+00 \\
-0.949136366576175 D+\infty\end{array}$ & $\begin{array}{l}0.546700049298597 D-14 \\
0.546500629691435 D-14\end{array}$ & $0.110-12$ & $0.290+01$ \\
\hline
\end{tabular}

Tables 3-1 and 3-2 show the values $(=R(I))$ for the left side of (4) and the number of iterations $(=N)$ required to obtain the approximations. The differences in these tables depend on the convergence rates of the methods used. The order of convergence is quadratic for Durand-Kerner's method, and cubic for Aberth's method. Some of the numerical results obtained by these methods are given in Tables 4, 5 and 6, where the underlined digits represent the incorrect digits, the upper values are derived from $A\left(x_{k}\right)$ and the lower values from $B\left(x_{k}\right)$. These results indicate that criterion (3) is effective in solving for complex zeros, and also applicable to polynomials with complex coefficients. 
TABLE 5

Numerical solutions obtained by Aberth's method

\begin{tabular}{|c|c|}
\hline No. & Real part \\
\hline \multirow[t]{2}{*}{1} & $\begin{array}{l}0.1250004281414680+02 \\
0.1250004743291180+02\end{array}$ \\
\hline & 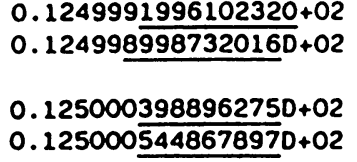 \\
\hline \multirow[t]{4}{*}{2} & $\begin{array}{l}0.122999999999219 D+01 \\
0.123000000000814 D+01\end{array}$ \\
\hline & $\begin{array}{l}0.121000000002556 \mathrm{D}+01 \\
0.120999999991999 \mathrm{D}+01\end{array}$ \\
\hline & $\begin{array}{l}0.119999999995365 D+01 \\
0.119999999997039 D+01\end{array}$ \\
\hline & $\begin{array}{l}0.121999999987617 D+01 \\
0.121999999987573 D+01\end{array}$ \\
\hline
\end{tabular}

$50.150000000000000 D+01$ $0.150000000000000 D+01$

$0.1500000000000000+01$ $0.150000000000000 D+01$

$0.5000000000000000+00$ $0.500000000000000 D+\infty 0$

$-0.1000000000000000+01$ $-0.1000000000000000+01$

$-0.100000000000000 D+01$ $-0.1000000000000000+01$

$0.500000000000000 D+00$ $0.5000000000000000+00$

$0.150000000000000 D+01$ $0.1500000000000000+01$

$0.1104536101718720+02$ $0.11045361017186 \bar{B} D+02$

$0.1109053650640950+02$

$0.110905365064101 \underline{D} D+02$

$-0.1000000000000000+01$

$-0.1000000000000000+01$ imations
$0.7381209548250390-04$
$0 . \overline{8872487838313480}-04$
$0.184431153368532 D-05$
$-0 . \overline{3467209592659070-05}$
$-0.7135300044845970-04$
$-0 . \overline{8669009136590400}-04$
$0.580-04 \quad 0.480+01$
$0.120-03 \quad 0.300+01$
$0.670-04 \quad 0.100+01$

$0.279324998809806 \mathrm{D}-20$

$0 . \overline{690664145973954 D}-20$

$-0.257050809038937 D-16$

$0 . \overline{258927384063799 D}-17$

$0.825241907992357 D-20$

$0 . \overline{199093843141447} \mathrm{D}-19$

$0.249328383289040 D-16$

$0 . \underline{2266481495840200}-16$

$0.155096364853693 D-24$

$-0.361891517991950 \mathrm{D}-24$

$0.1322875655532300+01$

$0.1322875655532300+01$

$0.8660254037844390+00$

$0.8660254037844390+\infty$

$0.100000000000000 D+01$ $0.1000000000000000+01$

$-0.100000000000000 D+01$ $-0.100000000000000 D+01$

$-0.8660254037844390+\infty 0$ $-0.8660254037844390+\infty$

$-0.132287565553230 \mathrm{D}+01$ $-0.1322875655532300+01$

$0.1109053650640940+02$ $0.110905365064090 D+02$

$0.1113552872566010+02$ $0.111355287256606 \bar{D}+02$

$0.1000000000000000+01$ $0.1000000000000000+01$
$S(I) \quad R(I)$

$0.150-09 \quad 0.120+02$

$0.10 D-15 \quad 0.54 D+08$

$0.15 D-09 \quad 0.12 D+02$

$0.44 D-09 \quad 0.12 D+02$

$0.16 D-14 \quad 0.15 D+10$

$0.78 D-16 \quad 0.45 D+02$

$0.14 D-15 \quad 0.83 D+01$

$0.00 D+\infty 00.10 D+51$

$0.000+\infty 00.200+51$

$0.000+\infty 00.100+51$

$0.780-16 \quad 0.45 D+02$

$0.130-120.160+02$

$0.780-120.130+01$

$0.000+00 \quad 0.100+51$ 


$$
\begin{gathered}
\text { TABLE } 6 \\
\text { Numerical solutions for complex coefficients }(\text { Aberth's method }) \\
f(x)=x^{3}-(9.424777961+8.154845485 i) x^{2}+(7.441644906+51.23840534 i) x \\
+38.63393639-60.39956197 i
\end{gathered}
$$$$
\text { Approximations }
$$
Real part
Imaginary part
$S(I)$
$R(I)$
$0.314439322215066 \mathrm{D}+01$
$0.2717955293101700+01$
$0.450-09$
$0.190+02$
$0.314439322214661 \mathrm{D}+01$
$0.271795529293159 \mathrm{D}+01$
$0.314047421908468 \mathrm{D}+01$
$0.2720869530552220+01$
$0.130-08$
$0.55 D+01$
0.314047421 913650D+01
$0.272086953050768 \mathrm{D}+01$
$0.313991052022448 \mathrm{D}+01$
$0.271602066176841 \mathrm{D}+01$
$0.13 D-08$
$0.58 D+01$
$0.313991052021273 \mathrm{D}+01$
$0.271602066186826 \mathrm{D}+01$

Generally, it is not a good idea to separate (3) into its real and imaginary parts, since if $x_{k}$ approaches a real zero, then underflow frequently occurs in the imaginary part.

Mathematical Laboratory

College of Agriculture and Veterinary Medicine

Nihon University

1866 Kameino Fujisawa-City

Kanagawa 252 Japan

1. S. Yamashita \& S. SATAKE, "On the calculation limit of roots of algebraic equations," Information Processing in Japan., v. 7, 1967, pp. 18-23.

2. S. Hirano, Numerical Solution of Algebraic Equations by Floating-Point Arithmetic, Thesis, Nihon Univ., 1980.

3. G. Peter \& J. H. Wilkinson, "Practical problems arising in the solution of polynomial equations," J. Inst. Math. Appl., v. 8, 1971, pp. 16-35.

4. M. Igarashi, "Zeros of polynomials and an estimation of its accuracy," J. Inform. Process., v. 5, 1982. pp. $172-175$.

5. I. O. Kerner, "Ein Gesamtschrittverfahren zur Berechnung der Nullstellen von Polynomen," Numer. Math., v. 8, 1966, pp. 290-294.

6. O. АвERTH, "Iteration methods for finding all zeros of a polynomial simultaneously," Math. Comp., v. 27,1973 , pp. $339-344$.

7. B. T. SMITH, "Error bounds for zeros of a polynomial based upon Gerschgorin's theorem," J. Assoc. Comput. Mach., v. 17, 1970, pp. 661-674. 